\title{
The Availability of Prebiotics, Probiotics and Other Gluten Free Natural Sources such as Millet in the Saudi Market to Enhance Celiac Patients' Quality of Life - A Descriptive Study in Jeddah, Saudi Arabia, 2017
}

\author{
Noura M S Eid*, Afaf Alharbi, Fatimah Al-shaiban, Mona Alajlani, Rawan Alghamdi \\ Faculty of Applied Medical Sciences, Department of Clinical Nutrition, King Abdulaziz University, Jeddah, Saudi Arabia \\ *Corresponding author: ooaeid2@kau.edu.sa
}

\begin{abstract}
The prevalence of celiac disease in Saudi Arabia has been increasing for 2.2\% of the Saudi population. Yet celiac disease (CD) patients are still suffering from long life gluten free diet GFD. According to literature, patients' diet can be improved by adding functional foods such as, prebiotics, probiotics and/or natural sources such as Millet to enhance the gut ecology and colon health. In the current study we have carried a descriptive study to assess the availability, prices and categories of food products suitable for celiac patients (probioitcs, prebiotics, gluten free products and millet as a natural source) in 6 main supermarkets, in Jeddah, Saudi Arabia, using a survey. Results showed that a higher costs of gluten free products seen in 6 main markets, except in "panda hypermarket". With regards to availabilities, the highest proportion of products was found in "Carrefour" and "Danube", have shown the highest, followed by "Lulu hypermarket" and "Manuel hypermarket" $(\mathrm{P}<0.05)$. The lowest amounts of gluten free products were seen in Farm superstores and Al Raya compared to others $(\mathrm{P}<0.05)$. With regards to categories were also looked at which shows Danube has most variations from staple, snacks, drinks, and additives. Millet has been seen to be available and lower in cost which is mostly suitable for patients at any economic level state. Findings suggest that the limited availability and high costs can influence celiac patients' quality of life and severity of symptoms among the Saudi populations. The current study has been carried for the first time in KSA, which recommends more local food production suitable for celiac patients and introducing the use of local natural sources such as millet to be used as staple food to enhance gut ecology health and reduce severity of celiac patients symptoms.
\end{abstract}

Keywords: celiac, prebiotic, probiotic, functional food, gluten free, supermarket

Cite This Article: Noura M S Eid, Afaf alharbi, Fatimah Al-shaiban, Mona alajlani, and Rawan al ghamdi, "The Availability of Prebiotics, Probiotics and Other Gluten Free Natural Sources such as Millet in the Saudi Market to Enhance Celiac Patients' Quality of Life - A Descriptive Study in Jeddah, Saudi Arabia, 2017." Journal of Food and Nutrition Research, vol. 6, no. 3 (2018): 187-191. doi: 10.12691/jfnr-6-3-8.

\section{Introduction}

The incidence of celiac disease is increasing, with considerable geographical differences, where it affects 0.6 to $1.0 \%$ of the population worldwide and with a ratio of female-to-male is 2.8:1. [1]. At geographical differences level, celiac disease in Palestinian populations who lived in a clarified area of Kuwait was three times more widespread. In Israel, celiac disease is more common in Jews compared with Arabs [2]. The prevalence of celiac disease in Saudi Arabia is $2.2 \%$ according to Saudi celiac patients support group.[3]. A study that shown prevalence among three regions of Saudi Arabia in difference location (southwestern - Western - center), where the highest celiac disease prevalence among the three studied regions in Saudi Arabia was in the Al- Qaseem region (3.1\%).
The prevalence by region was as follows: Aseer 2.1\% (10/479), Madinah 1.8\% (8/436), and Al- Qaseem 3.2\% (8/252). The prevalence in Aseer and Al-Qaseem were higher significantly than prevalence in Al Madinah [4]. There are several risk factors that can cause celiac disease, which include genetics. It can affect 1 in 10 people who are at first degree relative of the celiac patient, which is seen with parents or siblings at any age or race. When patients having one of these genes (HLA-DQ2 and HLA-DQ8) patients become at high risk for developing celiac disease. When ingestion of gluten protein, gliadin and glutenin results in activation of immune system, where they resist to be broken down with gastric acid. Accordingly, it damages the intestinal mucosal villi and lead to GI irritability like chronic diarrhea, flatulence, gasses, constipation, fatigue, weight loss, bloating, anemia, and nutrients malabsorption especially fat, calcium, iron, and folate [5]. The main nutritional therapy is a gluten free 
diet (GFD), which is a diet in which gluten is removed in particular gliadin, which relieves the symptoms of the disease [6]. Gluten free diet can be found in many sources meat, fish, fruit and vegetables, rice, potatoes, and lentils.[7]. However purchasing gluten free products can be expensive and restrictive which affect the life-long therapy [8]. Its may have a significant contrary effect upon the quality of life of the patients. Furthermore, gluten free diet is also free from some of essential nutrients such as vitamins and minerals supplements. Thus, it is suggested to find an alternative which is naturally available such as millet, amaranth, soybean, and potato [9]. Furthermore, the daily amount of tolerable gluten is in constant among people but consumption of less than $10 \mathrm{mg}$ of gluten per day has been stated to be safe for patients with celiac disease. A cross-sectional study was conducted in which an online questionnaire to all families registered in the Saudi celiac patients support group, showing a significant negative socio-economic impact of GFD on children with CD \& their families [10].

Recent research in diet and nutrition has been directed towards the gut microbial state in the human body. Microbiome is referred to billions of living microorganism, which is estimated to be more than 3000 strains found in the intestines such as Bifidobacterium, lactobacillus, Bacteroides, and many others that ferment carbohydrates to making short chain fatty acids (SCFA) such as butyrate, propionate and acetate, encountering protective, structural, and metabolic functions in the colon, and that's why it is the most metabolically active organ in the human body [11]. According to the literature celiac disease patients have lower numbers of Bifidobacterium spp., while the number of Bacteroides spp. is found to be higher in stools and duodenal biopsies of celiac disease patients whether they are treated or untreated with a GFD compared to healthy individuals [12]. Several studies in children have also reported increased prevalence of Bacteroidesvulgatus and $E$. coli in celiac disease biopsies before and after GFD [13]. The state of the gut microbiota plays crucial roles in immunity and severity of symptoms. However, introducing functional foods such as prebiotics and probiotics are believed to reduce severity of symptoms and enhance quality of life [14]. Probiotics are defined as " "Live microorganisms which, when administered in adequate amounts confer a health benefit on the host"), such as lactobacillus spp., bifidobacterium spp., and bacillus, which are found in the market as supplements, dairy products, dry soups, baked goods and others (FAO/WHO Expert Consultation Report, 2001. Whereas prebiotics are defined as "a substrate that is selectively utilized by host microorganisms conferring a health benefit. This definition expands the concept of prebiotics to possibly include non-carbohydrate substances, applications to body sites other than the gastrointestinal tract, and diverse categories other than food" by Glenn Gibson 2017 [15] They have been classified based on their structure into two groups, the Inulin-type fructans (ITF) and Galacto-oligosaccharides (GOS). Prebiotics may have some benefits not only in the colon but also in the oral cavity, as well as on the skin, they can be found naturally in food such as leeks, banana, onion, asparagus, and garlic.
Millet is one of the cereal group shaped as very small circles, it considered as an ancient seed found in numerous amounts in India and parts in Africa, eaten by humans and animals. It can be used for multiple cooks. More interestingly it is a gluten-free grain which is an excellent choice for people who are suffering from celiac disease. It is also a great source for macronutrient and micronutrients. Millet has been proved to exhibit a prebiotic action for diarrhea in young children. [16]. There are many types of millet, most popular are Pearl millet, Finger millet, and Foxtail millet. Studies have shown that pearl millet is characterized with anti-diabetic properties and alleviate constipation that celiac patients may experience. Furthermore, Finger millet is characterize with unique proteins like tryptophan, and cysteine [17] and Foxtail millet has been proved to have a hypoglycemic effect due to high content of complex carbohydrates and resistant starch. [18]. Some beneficial actions that prebiotics can perform are reducing inflammation, improving symptoms associated with IBD, enhancing the bioavailability and uptake of minerals including calcium, magnesium and possibly iron, and promoting satiety to reduce weight and prevent obesity [19].

Thus, availability, prices and categories of gluten free and functions food products plays major roles in supporting celiac patients diet and reducing severity of their symptoms. There is a lack of information related to the existence of such products, where we have not found one study carried in Saudi Arabia covering this case. The current study objectives are to assess the availability, process and categories of gluten free products, prebiotics, probiotics and Millet in the main supermarkets in Jeddah, Saudi Arabia, to provide a descriptive information for physicians and clinical dietitians to be able to apply effective practice and realistic recommendations which suits all patients.

\section{Materials and Methods}

\subsection{Study Design}

A descriptive study was carried on 6 main supermarkets in Jeddah; Danube, Al-Raya, Hyper Panda, lulu hypermarket, farm superstore, Carrefour, and Manuel) to assess the availability and prices of gluten free products, prebiotics, probiotics and millet. Analysis has been analyzed descriptively and categorized as type of product, amount, and price. study protocol was approved by Medicine College Ethics Committee at King AbdulAziz university (Reference HA-02-J-008).

\subsection{Statistical Analysis}

Data were analyses using SPSS software (version 21). A one-way ANOVA was performed to test the difference in price between celiac disease products. Non-parametric binomial test was performed to test the difference in celiac disease proportions between Jeddah hypermarkets. A Two-Sided P values of 0.05 or less were considered statistically significant. Data were represented as mean $\pm \mathrm{SE}$. 


\section{Results \& Discussion}

\subsection{Availability and Food Categories of Prebiotics, Probiotics, and Gluten Free Types of Foods}

The current study was carried for the first time in Saudi Arabia, to detect the availability of probiotics, prebiotics and gluten free functional foods in Jeddah supermarkets to support dietitians and nutritionists' roles in food recommendations for celiac patients in the country. Results have shown that prebiotics do not exist in any of the supermarkets, whereas only probiotics are found and only added to yogurts, which include products like (Activia and Vetal). On the other hand, gluten free products have been found in all supermarkets except in "panda hypermarket". "Carrefour" and "Danube", have shown the highest proportion of products suitable for celiac patients, followed by "Lulu hypermarket" and "Manuel hypermarket" ( $>0.05)$. The lowest amounts of gluten free products were seen in Farm superstores and Al Raya compared to others $(\mathrm{P}<0.05)$ (Table 1). Similar data has seen in UK that might be due to defect in labelling, which could be a factor of not representing the actual number of available product [20]. In India findings has stated no availability of gluten free food products, which could be due to the lower demand on those products [21]. We assume that the higher the demand on products, leads to the higher the availability. Availability of certain food products have a high impact on the dietary intakes of celiac patients. A prospective longitudinal population study assessed Sweden populations and how available food products can solve problems associated with popular food products, such as bread, where the existence of gluten free bread covers the carbohydrate food group [22]. Furthermore, availabity of prebiotics and probiotics redce severity of symptoms. A randomized placebo controlled trial have tested the impact of oligo fructose following a gluten free diet on 34 pediatric celiac patients, have shown to increase short chain fatty acids by $31 \%$ in comparison with baseline, which plays major roles in the gut health and reducing symptoms.[23]. In addition the availability of millet flour is considered supportive for the celiac populations due to the presence of polyphenols and dietary fibres exhibiting several protective actions in the colon and [24] both are considered to exhibit a prebiotic potential [6]. Food categories also varied between hypermarkets, which include staple, snacks, drinks, and additives, which are the main concerns of patients. These information are believed to support the role of dietitians in nutrition therapy. Results has shown that gluten free products are mostly staple foods, such as bread, pasta and cereal and snacks, such as crackers and biscuits found in high amounts in Carrefour and Danube in comparison with Manuel hypermarket $(\mathrm{P}<0.05)$. Whereas Al Raya had only 3 types of snacks include: Digestive biscuit, corn thins (multi grain), corn thins (sesame), which does not support a daily diet, but could be used in occasions such as birthdays and family events. With regards to snacks it has been seen mostly in Danube but significantly lower products in Lulu hypermarket $(\mathrm{P}<0.05)$. The snacks proportion in Farm superstores and Al Raya supermarket is significantly lower than other hypermarkets $(\mathrm{P}<0.05)$. There are no significant differences in drink proportions between hypermarkets in Jeddah, where the main producers found are in lulu hyper market, Danube, and AlRaya. Study has also looked at. There are no significant differences in additive proportions between Manuel, Danube and Lulu hypermarkets and an absence of additive products were seen in Carrefour, Farm superstores and Al Raya super markets. Among all categories, the most useful is the staple type, which replace several important food products in the daily diet. According to a retrospective review of diet history records by dietitians, replacing flour with an alternative gluten free flour have increased levels of protein, iron, calcium and fibre [25].

\subsection{Food Costs of Prebiotics, Probiotics and Gluten Free Types of Foods}

Gluten free market has expanded in the past years mainly in the developed world, such as UK and US, with different nutrition values, in comparison with other food products, such as higher amount of fat and also it has been confirmed that it is significantly in higher costs too [26]. In the current study, we have looked at different costs (Figure 1), where results have demonstrated that cost of drinks were significantly lower than snacks, staple foods and additives. Additive prices were significantly lower than snack price. High costs with limited availability can influence those with less mobility such as elderly and disabled people especially with low socioeconomic status resulting in poor adherence to GFD which can lead to villous atrophy and other complications[27]. The reasons behind higher costs of GF products are the processing procedures that the product go through and removal of gluten [28]. With regards to our study, most of the products present are imported, which adds to the expense of the original product price. According to the KACST report on biotechnology, KSA accounts for small publication in the field of biotechnology and nearly no collaborations in producing new products with other countries, in comparison with the Netherland, USA, and the UK [29].

Table 1. The variety and number of type of foods in several supermarkets

\begin{tabular}{|c|c|c|c|c|c|c|c|}
\hline & $\begin{array}{l}\text { Manuel } \\
\mathrm{n}(\mathrm{N} \%)\end{array}$ & $\begin{array}{l}\text { Carrefour } \\
\mathrm{N}(\mathrm{N} \%)\end{array}$ & $\begin{array}{c}\text { Farm superstores } \\
\mathrm{N}(\mathrm{N} \%)\end{array}$ & $\begin{array}{l}\text { Al Raya } \\
\text { N (N \%) }\end{array}$ & $\begin{array}{l}\text { Danube } \\
\mathrm{n}(\mathrm{N} \%)\end{array}$ & $\begin{array}{c}\text { Lulu } \\
\mathrm{n}(\mathrm{N} \%)\end{array}$ & Total \\
\hline Staple & $8(9.9 \%)^{b}$ & $36(44.4 \%)^{\mathrm{a}}$ & $6(7.4 \%)^{b}$ & $0(0 \%)^{\mathrm{c}}$ & $16(19.8 \%)^{b}$ & $15(18.5 \%)^{b}$ & $81(100.00 \%)$ \\
\hline Snack & $13(15.9 \%)^{b}$ & $16(19.5 \%)^{b}$ & $2(2.4 \%)^{\mathrm{c}}$ & $3(3.7 \%)^{c}$ & $31(37.8 \%)^{\mathrm{a}}$ & $17(20.7 \%)^{\mathrm{a}, \mathrm{b}}$ & $82(100.00 \%)$ \\
\hline Drink & $0(0 \%)$ & $0(0 \%)$ & $0(0 \%)$ & $1(25.00 \%)$ & $1(25.00 \%)$ & $2(50.00 \%)$ & $4(100.00 \%)$ \\
\hline Additive & $3(25.0 \%)^{\mathrm{a}, \mathrm{b}}$ & $0(0 \%)^{b}$ & $0(0 \%)^{b}$ & $0(0 \%)^{b}$ & $3(25.0 \%)^{\mathrm{a}, \mathrm{b}}$ & $6(50.0 \%)^{a}$ & $12(100.00 \%)$ \\
\hline Total & $24(13.4 \%)^{b}$ & $52(29.1 \%)^{\mathrm{a}}$ & $8(4.5 \%)^{c}$ & $4(2.2 \%)^{c}$ & $51(28.5 \%)^{\mathrm{a}}$ & $40(22.3 \%)^{\mathrm{a}, \mathrm{b}}$ & $179(100.00 \%)$ \\
\hline
\end{tabular}




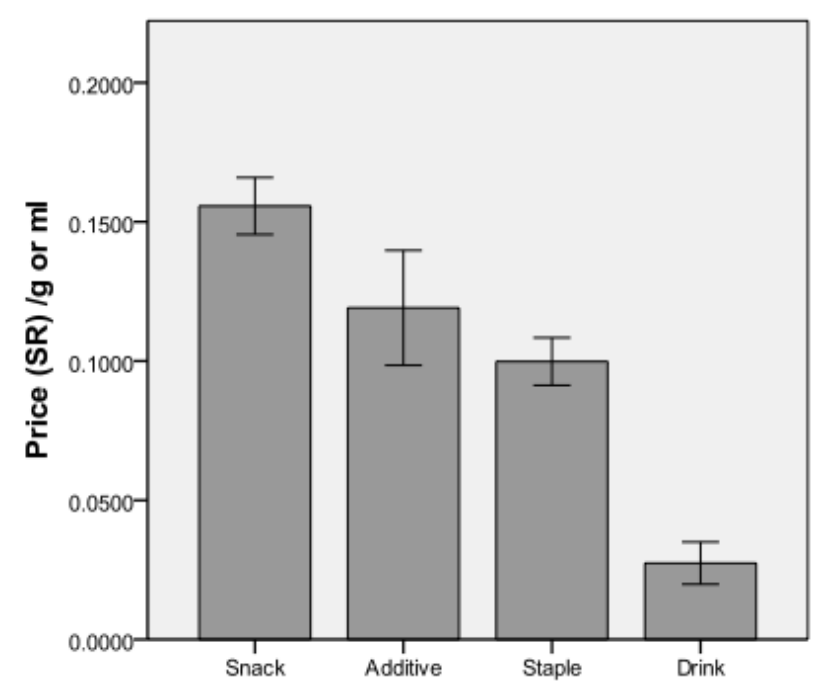

Figure 1. Comparison between the prices of (staple, snacks, drinks, additives)/g or $\mathrm{ml}$ in the 7 main supermarkets

\subsection{The Availability of Millet Flour as an Alternative}

The current study also covers research on possible alternative, which is usually seen as staple foods, such as millet flour. According to our data, staple food prices were significantly lower than additives and snacks and that include millet flour. Millet can be used for several food products that can be prepared at home, such as baking doughs i.e. breads and cakes and also can be used to prepare pasta and cereal [30]. It is a gluten-free grain which is an excellent choice for people who suffering from celiac disease, found in many grinders in the form of flour with low price similar to wheat flour (Figure 2), and locally grown in Saudi Arabia in higher amounts in Gazan [31]. According to several trials, millet is considered dietary ingredients which can lower symptoms of celiac patients due to antioxidant actions, due to the presence of phenolic acids, such as vanillic acid, syringic acid, $p$-coumaric acid and ferulic acid [32].

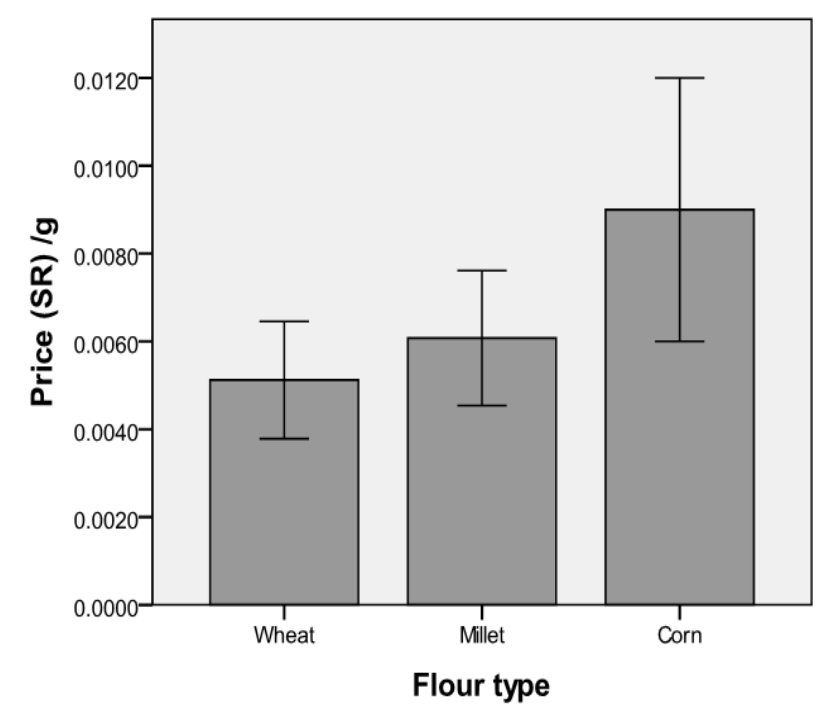

Figure 2. The different types of flour and there is no significant difference in the price between them.

\section{Conclusion}

In conclusion, our descriptive data has been conducted on all available food products suitable for celiac patients, including their categories and prices, which are found in 6 main supermarkets in Jeddah including their categories and prices, which shows limited availability of gluten free products (millet, pre- biotics\& pro-biotics) and the small amount present is at a higher cost can. This is believed to influence celiac patients' quality of life, severity of symptoms and the statistical increase of incidence in KSA. Another aspect is the the possible lack of awareness in celiac patients about the functional foods and its impact on the gut health which results on low demand more studies are needed in the production and import of probiotic and prebiotics in KSA. and more trials are needed towards alternatives such as millet to be used as staple food and its impact on the gut ecology and symptoms of celiac patients. Even though gluten free product are significantly expensive [33], natural sources of gluten free flour like, millet have an excellent choice for people who suffering from celiac disease and also its cost significantly lower compared to others products and can used in multiple cooks as in pastry products i.e. breads, pastas, and cakes as well. We recommended to establish new policies for grinders from food and drug administration regarding cross contamination that could happened during separation process with other gluten free flours and increase production lines in food industries regarding gluten free products thus cost of gluten free products would be declined and become more available, with assigning fully trained staff including dietitians in marketing area to check clear food label, food safety and analysis, finally role of dietitians are crucial in innovate new gluten free products and teaching low socioeconomic people for other alternative methods instead of purchasing costly ones.

\section{Acknowledgments}

We would like to express our sincere appreciation and gratitude to the managers of the supermarkets for their collaborations and their time to support our research

\section{References}

[1] Rafael Parra-Medina, N. M.-G., . Prevalence of Celiac Disease in Latin America: A Systematic Review and Meta-Regression. PLOS (2015).

[2] J. Y. Kang, A. H. Y. K., A. Green,. Systematic review: worldwide variation in the frequency of coeliac disease and changes over time. Alimentary Pharmacology \& Therapeutics, 38, 3 (2013).

[3] Available at http://www.saudiceliac.com/en. City.

[4] Abdulrahman M Aljebreen, M. A. A., Alwaleed Alhammad, Faleh Z Al Faleh Seroprevalence of celiac disease among healthy adolescents in Saudi Arabia World journal of gastroenterology 19, 15 (April 21, 2013).

[5] Celiac, B. celiac disease risk factors available at https://www.beyondceliac.org/celiac-disease/risk-factors/. City, 2016.

[6] Tuohy, K. M., Conterno, L., Gasperotti, M. and Viola, R. Upregulating the Human Intestinal Microbiome Using Whole Plant Foods, Polyphenols, and/or Fiber. Journal of Agricultural and Food Chemistry, 60, 36 (2012/09/12 2012), 8776-8782. 
[7] L. Kathleen Mahan MS RD CDE (Author), J. L. R. M. R. C. A. Sylvia Escott-Stump MA RD LDN Krause's Food \& the Nutrition Care Process, 13th Edition (2004).

[8] Laurikka, P. Gastrointestinal Symptoms in Celiac Disease Patients on a Long-Term Gluten-Free Diet, 8, 7 (2016).

[9] Shahrooz, R. J., A, M,. Review Article: Celiac Disease, New Approaches to Therapy, 35, 7 (2012), 768-781.

[10] Ahmed Sarkhy, M. I. E. M., Elshazaly Saeed,. Socioeconomic Impacts of Gluten-Free Diet among Saudi Children with Celiac Disease. Pediatric Gastroenterology Hepatology \& Nutrition, 19, 3 (2016 Sep)

[11] M., C. Role of the gut microbiota in health and chronic gastrointestinal disease: understanding a hidden metabolic organ. Therapeutic Advances Gastroenterology 6, 4 (2013), 295-308.

[12] Cenit, M. C., Olivares, M., Codoñer-Franch, P., \& Sanz, Y. Intestinal Microbiota and Celiac Disease: Cause, Consequence or Co-Evolution? National Institutes of Health, 7, 8 (2015), 6900-6923.

[13] Di Cagno R, D. A. M., De Pasquale I, Ndagijimana M, Vernocchi P, Ricciuti P, Gagliardi F, Laghi L, Crecchio C, Guerzoni ME, Gobbetti M, Francavilla R. Duodenal and faecal microbiota of celiac children: molecular, phenotype and metabolome characterization. Bio Med Central Journal (Oct 2011).

[14] Conlon, M., Bird, Anthony The Impact of Diet and Lifestyle on Gut Microbiota and Human Health. Nutrients (2015), 2072-6643.

[15] Gibson, G. R., Hutkins, R., Sanders, M. E., Prescott, S. L., Reimer, R. A., Salminen, S. J., Scott, K., Stanton, C., Swanson, K. S., Cani, P. D., Verbeke, K. and Reid, G. Expert consensus document: The International Scientific Association for Probiotics and Prebiotics (ISAPP) consensus statement on the definition and scope of prebiotics. Nature reviews. Gastroenterology \& hepatology, 14, 8 (Aug 2017), 491-502.

[16] Issoufou Amadou, M. E. Millets: Nutritional composition, some health benefits and processing. Emirates Journal of Food and Agriculture, 25 7(2013), 501-508.

[17] Sadhguru 7 Health Benefits of Ragi \& 6 Easy Ragi Recipes availabe at http://isha.sadhguru.org/blog/lifestyle/food/7-healthbenefits-of-ragi-6-great-ragi-recipes/. City.

[18] María de Lourdes Moreno, I. C. a. C. S. Alternative Grains as Potential Raw Material for Gluten- Free Food Development in The Diet of Celiac and Gluten- Sensitive Patients. Austin Journal of Nutrition and Food Sciences, 2, 3 (March 13, 2014).

[19] Amy M. Brownawell, W. C., Glenn R. Gibson,. Prebiotics and the Health Benefits of Fiber: Current Regulatory Status, Future Research, and Goals. journal of Nutrition (2012).

[20] Singh, J. and Whelan, K. Limited availability and higher cost of gluten-free foods. Journal of Human Nutrition and Dietetics, 24, 5 (2011), 479-486.

[21] Rajpoot, P. Problems and Challenges to Adaptation of Gluten Free Diet by Indian Patients with Celiac Disease. Nutrients (2013), 4869-4879.
[22] Kautto, E., Rydén, P. J., Ivarsson, A., Olsson, C., Norström, F., Högberg, L., Carlsson, A., Hagfors, L. and Hörnell, A. What happens to food choices when a gluten-free diet is required? A prospective longitudinal population-based study among Swedish adolescent with coeliac disease and their peers. Journal of Nutritional Science, 3 (2014), e2.

[23] Drabinska, N., Jarocka-Cyrta, E., Markiewicz, L. H. and KrupaKozak, U. The Effect of Oligofructose-Enriched Inulin on Faecal Bacterial Counts and Microbiota-Associated Characteristics in Celiac Disease Children Following a Gluten-Free Diet: Results of a Randomized, Placebo-Controlled Trial. Nutrients, 10, 2 (Feb 12 2018).

[24] Devi, P. B., Vijayabharathi, R., Sathyabama, S., Malleshi, N. G. and Priyadarisini, V. B. Health benefits of finger millet (Eleusine coracana L.) polyphenols and dietary fiber: a review. Journal of Food Science and Technology, 51, 6 (2014/06/01 2014), 1021-1040.

[25] Lee, A. R., Ng, D. L., Dave, E., Ciaccio, E. J. and Green, P. H. R. The effect of substituting alternative grains in the diet on the nutritional profile of the gluten-free diet. Journal of Human Nutrition and Dietetics, 22, 4 (2009), 359-363.

[26] Fry, L., Madden, A. M. and Fallaize, R. An investigation into the nutritional composition and cost of gluten-free versus regular food products in the UK. Journal of Human Nutrition and Dietetics, 31, 1 (2018), 108-120.

[27] Burden, M., Mooney, P. D., Blanshard, R. J., White, W. L., Cambray-Deakin, D. R. and Sanders, D. S. Cost and availability of gluten-free food in the UK: in store and online. Postgraduate Medical Journal, 91, 1081 (2015), 622-626.

[28] Papageorgiou, M. and Skendi, A. 1 - Introduction to cereal processing and by-products A2 - Galanakis, Charis M. Woodhead Publishing, City, 2018.

[29] Al-Swailem, A. M. Strategic Priorities for Biotechnology Program. KACST (2014).

[30] Chakraborty, S. K., Kotwaliwale, N. and Navale, S. A. Rheological characterization of gluten free millet flour dough. Journal of Food Measurement and Characterization (2018/02/10 2018).

[31] Basahy, A. Y. Nutritional and chemical evaluation of pearl millet grains (Pennisetum typhoides (Burm. f.) Stapf \& Hubbard, Poaceae) grown in the Gizan area of Saudi Arabia. International journal of food sciences and nutrition, 47, 2 (Mar 1996), 165-169.

[32] Guo, X., Sha, X., Rahman, E., Wang, Y., Ji, B., Wu, W. and Zhou, F. Antioxidant capacity and amino acid profile of millet bran wine and the synergistic interaction between major polyphenols. J Food Sci Technol, 55, 3 (Mar 2018), 1010-1020.

[33] Rajpoot, P. and Makharia, G. K. Problems and challenges to adaptation of gluten free diet by Indian patients with celiac disease. Nutrients, 5, 12 (Nov 27 2013), 4869-4879. 